\title{
Conditional intuitionistic fuzzy probability and martingale convergence theorem using IF-probability
}

\author{
Katarína Čunderlíková \\ Mathematical Institute, Slovak Academy of Sciences \\ Štefánikova 49, 81473 Bratislava, Slovakia \\ e-mail: cunderlikova. lendelova@gmail.com
}

Received: 15 May $2020 \quad$ Revised: 25 September $2020 \quad$ Accepted: 27 September 2020

\begin{abstract}
The aim of this paper is to formulate the conditional intuitionistic fuzzy probability and a version of martingale convergence theorem with respect an intuitionistic fuzzy probability. Since the intuitionistic fuzzy probability can be decomposed to two intuitionistic fuzzy states, we can use the results holding for intuitionistic fuzzy states.

Keywords: Intuitionistic fuzzy event, Intuitionistic fuzzy observable, Intuitionistic fuzzy state, Intuitionistic fuzzy probability, Product, Conditional intuitionistic fuzzy probability, Martingale convergence theorem.
\end{abstract}

2010 Mathematics Subject Classification: 03B52, 60A86, 60G48.

\section{Introduction}

In $[1,2]$ K. T. Atanassov introduced the notion of intuitionistic fuzzy sets. Later, P. Grzegorzewski and E. Mrówka defined the probability on the family of intuitionistic fuzzy events

$$
\mathcal{N}=\left\{\left(\mu_{A}, \nu_{A}\right) ; \mu_{A}, \nu_{A} \text { are } \mathcal{S}-\text { measurable and } \mu_{A}+\nu_{A} \leq 1_{\Omega}\right\}
$$

as a mapping $\mathcal{P}$ from the family $\mathcal{N}$ to the set of all compact intervals in $R$ by the formula

$$
\mathcal{P}\left(\left(\mu_{A}, \nu_{A}\right)\right)=\left[\int_{\Omega} \mu_{A} d P, 1-\int_{\Omega} \nu_{A} d P\right],
$$


where $(\Omega, \mathcal{S}, P)$ is probability space, see [5]. This intuitionistic fuzzy probability was axiomatically characterized by B. Riečan (see [8]).

Later in [6] K. Lendelová defined the conditional intuitionistic fuzzy probability only for separating intuitionistic fuzzy probability and she showed their properties in this case.

In [13] B. Riečan introduced the conditional intuitionistic fuzzy probability $\mathbf{p}(\mathbf{A} \mid x)$ as a Borel measurable function $f$ (i.e. $B \in \mathcal{B}(R) \Longrightarrow f^{-1}(B) \in \mathcal{B}(R)$ ) such that

$$
\int_{B} \mathbf{p}(\mathbf{A} \mid x) d \mathbf{m}_{x}=\mathbf{m}(\mathbf{A} \cdot x(B))
$$

for each $B \in \mathcal{B}(R)$. There $\mathbf{A} \in \mathcal{F}, x: \mathcal{B}(R) \rightarrow \mathcal{F}$ is intuitionistic fuzzy observable and $\mathbf{m}$ is the intuitionistic fuzzy state defined by B. Riečan on the family of intuitionistic fuzzy events

$$
\mathcal{F}=\left\{\left(\mu_{A}, \nu_{A}\right) ; \mu_{A}+\nu_{A} \leq 1_{\Omega}\right\},
$$

where $\mu_{A}, \nu_{A}$ are $\mathcal{S}$-measurable functions, $\mu_{A}, \nu_{A}: \Omega \rightarrow[0,1]$, as a mapping $\mathbf{m}$ from the family $\mathcal{F}$ to the set $R$ by the formula

$$
\mathbf{m}\left(\left(\mu_{A}, \nu_{A}\right)\right)=(1-\alpha) \int_{\Omega} \mu_{A} d P+\alpha\left(1-\int_{\Omega} \nu_{A} d P\right),
$$

where $P: \mathcal{S} \rightarrow[0,1]$ is a probability measure and $\alpha \in[0,1]$ (see [10]).

In paper [4] we studied properties of the conditional intuitionistic fuzzy probability with respect to the intuitionistic fuzzy state and we formulated martingale convergence in this case. Since the intuitionistic fuzzy probability can be decomposed to two intuitionistic fuzzy states, we can use the results holding for intuitionistic fuzzy states. Therefore, we try to formulate the conditional intuitionistic fuzzy probability and a version of martingale convergence theorem with respect an intuitionistic fuzzy probability.

Remark that in a whole text we use a notation IF as an abbreviation for intuitionistic fuzzy.

\section{IF-event, IF-probability, IF-state and IF-observable}

In this section we explain the basic notions from IF-probability theory, see $[1,2,11-13]$.

Definition 2.1. Let $\Omega$ be a nonempty set. An IF-set $\mathbf{A}$ on $\Omega$ is a pair $\left(\mu_{A}, \nu_{A}\right)$ of mappings $\mu_{A}, \nu_{A}: \Omega \rightarrow[0,1]$ such that $\mu_{A}+\nu_{A} \leq 1_{\Omega}$.

Definition 2.2. Start with a measurable space $(\Omega, \mathcal{S})$. Hence $\mathcal{S}$ is a $\sigma$-algebra of subsets of $\Omega$. An IF-event is called an IF-set $\mathbf{A}=\left(\mu_{A}, \nu_{A}\right)$ such that $\mu_{A}, \nu_{A}: \Omega \rightarrow[0,1]$ are $\mathcal{S}$-measurable.

The family of all IF-events on $(\Omega, \mathcal{S})$ will be denoted by $\mathcal{F}, \mu_{A}: \Omega \longrightarrow[0,1]$ will be called the membership function, $\nu_{A}: \Omega \longrightarrow[0,1]$ be called the non-membership function.

If $\mathbf{A}=\left(\mu_{A}, \nu_{A}\right) \in \mathcal{F}, \mathbf{B}=\left(\mu_{B}, \nu_{B}\right) \in \mathcal{F}$, then we define the Lukasiewicz binary operations $\oplus, \odot$ on $\mathcal{F}$ by

$$
\begin{aligned}
& \left.\mathbf{A} \oplus \mathbf{B}=\left(\left(\mu_{A}+\mu_{B}\right) \wedge 1_{\Omega},\left(\nu_{A}+\nu_{B}-1\right) \vee 0_{\Omega}\right)\right) \\
& \left.\mathbf{A} \odot \mathbf{B}=\left(\left(\mu_{A}+\mu_{B}-1\right) \vee 0_{\Omega},\left(\nu_{A}+\nu_{B}\right) \wedge 1_{\Omega}\right)\right)
\end{aligned}
$$


and the partial ordering is given by

$$
\mathbf{A} \leq \mathbf{B} \Longleftrightarrow \mu_{A} \leq \mu_{B}, \nu_{A} \geq \nu_{B}
$$

Consider a probability space $(\Omega, \mathcal{S}, P)$. Then in [5] the IF-probability $\mathcal{P}(\mathbf{A})$ of an IF-event $\mathbf{A}=\left(\mu_{A}, \nu_{A}\right) \in \mathcal{F}$ has been defined as a compact interval by the equality

$$
\mathcal{P}(\mathbf{A})=\left[\int_{\Omega} \mu_{A} d P, 1-\int_{\Omega} \nu_{A} d P\right] .
$$

Let $\mathcal{J}$ be the family of all compact intervals. Then the mapping $\mathcal{P}: \mathcal{F} \rightarrow \mathcal{J}$ can be defined axiomatically similarly as in [8].

Definition 2.3. Let $\mathcal{F}$ be the family of all IF-events in $\Omega$. A mapping $\mathcal{P}: \mathcal{F} \rightarrow \mathcal{J}$ is called an IF-probability, if the following conditions hold:

(i) $\mathcal{P}\left(\left(1_{\Omega}, 0_{\Omega}\right)\right)=[1,1], \mathcal{P}\left(\left(0_{\Omega}, 1_{\Omega}\right)\right)=[0,0]$;

(ii) if $\mathbf{A} \odot \mathbf{B}=\left(0_{\Omega}, 1_{\Omega}\right)$, then $\mathcal{P}(\mathbf{A} \oplus \mathbf{B})=\mathcal{P}(\mathbf{A})+\mathcal{P}(\mathbf{B})$;

(iii) if $\mathbf{A}_{n} \nearrow \mathbf{A}$, then $\mathcal{P}\left(\mathbf{A}_{n}\right) \nearrow \mathcal{P}(\mathbf{A})$.

(Recall that $\left[\alpha_{n}, \beta_{n}\right] \nearrow[\alpha, \beta]$ means that $\alpha_{n} \nearrow \alpha, \beta_{n} \nearrow \beta$, but $\mathbf{A}_{n}=\left(\mu_{A_{n}}, \nu_{A_{n}}\right) \nearrow \mathbf{A}=$ $\left(\mu_{A}, \nu_{A}\right)$ means $\mu_{A_{n}} \nearrow \mu_{A}, \nu_{A_{n}} \searrow \nu_{A}$.)

IF-probability $\mathcal{P}$ is called separating, if

$$
\mathcal{P}\left(\left(\mu_{A}, \nu_{A}\right)\right)=\left[\mathcal{P}^{b}\left(\mu_{A}\right), 1-\mathcal{P}^{\sharp}\left(\nu_{A}\right)\right],
$$

where the functions $\mathcal{P}^{b}, \mathcal{P}^{\sharp}: \mathcal{T} \rightarrow[0,1]$ are probabilities.

Of course, each $\mathcal{P}(\mathbf{A})$ is an interval, denote it by $\mathcal{P}(\mathbf{A})=\left[\mathcal{P}^{b}(\mathbf{A}), \mathcal{P}^{\sharp}(\mathbf{A})\right]$. By this way we obtain two functions

$$
\mathcal{P}^{b}: \mathcal{F} \rightarrow[0,1], \mathcal{P}^{\sharp}: \mathcal{F} \rightarrow[0,1]
$$

and some properties of $\mathcal{P}$ can be characterized by some properties of $\mathcal{P}^{b}, \mathcal{P}^{\sharp}$, see [9].

Theorem 2.4. Let $\mathcal{P}: \mathcal{F} \rightarrow \mathcal{J}$ and $\mathcal{P}(\mathbf{A})=\left[\mathcal{P}^{b}(\mathbf{A}), \mathcal{P}^{\sharp}(\mathbf{A})\right]$ for each $\mathbf{A} \in \mathcal{F}$. Then $\mathcal{P}$ is an IF-probability if and only if $\mathcal{P}^{b}$ and $\mathcal{P}^{\sharp}$ are IF-states.

Proof. In [9, Theorem 2.3] .

Recall that by an intuitionistic fuzzy state (IF-state) $\mathbf{m}$ we understand each mapping $\mathbf{m}: \mathcal{F} \rightarrow[0,1]$ which satisfies the following conditions (see [10]):

(i) $\mathbf{m}\left(\left(1_{\Omega}, 0_{\Omega}\right)\right)=1, \mathbf{m}\left(\left(0_{\Omega}, 1_{\Omega}\right)\right)=0$;

(ii) if $\mathbf{A} \odot \mathbf{B}=\left(0_{\Omega}, 1_{\Omega}\right)$ and $\mathbf{A}, \mathbf{B} \in \mathcal{F}$, then $\mathbf{m}(\mathbf{A} \oplus \mathbf{B})=\mathbf{m}(\mathbf{A})+\mathbf{m}(\mathbf{B})$;

(iii) if $\mathbf{A}_{n} \nearrow \mathbf{A}$ (i.e. $\left.\mu_{A_{n}} \nearrow \mu_{A}, \nu_{A_{n}} \searrow \nu_{A}\right)$, then $\mathbf{m}\left(\mathbf{A}_{n}\right) \nearrow \mathbf{m}(\mathbf{A})$. 
Now we introduce the notion of an observable. Let $\mathcal{J}$ be the family of all intervals in $R$ of the form

$$
[a, b)=\{x \in R: a \leq x<b\} .
$$

Then the $\sigma$-algebra $\sigma(\mathcal{J})$ is denoted by $\mathcal{B}(R)$ and it is called the $\sigma$-algebra of Borel sets, its elements are called Borel sets (see [14]).

Definition 2.5. By an IF-observable on $\mathcal{F}$ we understand each mapping $x: \mathcal{B}(R) \rightarrow \mathcal{F}$ satisfying the following conditions:

(i) $x(R)=\left(1_{\Omega}, 0_{\Omega}\right), x(\emptyset)=\left(0_{\Omega}, 1_{\Omega}\right)$;

(ii) if $A \cap B=\emptyset$ and $A, B \in \mathcal{B}(R)$, then $x(A) \odot x(B)=\left(0_{\Omega}, 1_{\Omega}\right)$ and $x(A \cup B)=$ $x(A) \oplus x(B)$;

(iii) if $A_{n} \nearrow A$ and $A_{n}, A \in \mathcal{B}(R), n \in N$, then $x\left(A_{n}\right) \nearrow x(A)$.

Similarly we can define the notion of n-dimensional IF-observable.

Definition 2.6. By an n-dimensional IF-observable on $\mathcal{F}$ we understand each mapping $x: \mathcal{B}\left(R^{n}\right) \rightarrow \mathcal{F}$ satisfying the following conditions:

(i) $x\left(R^{n}\right)=\left(1_{\Omega}, 0_{\Omega}\right), x(\emptyset)=\left(0_{\Omega}, 1_{\Omega}\right)$;

(ii) if $A \cap B=\emptyset$ and $A, B \in \mathcal{B}\left(R^{n}\right)$, then $x(A) \odot x(B)=\left(0_{\Omega}, 1_{\Omega}\right)$ and $x(A \cup B)=$ $x(A) \oplus x(B)$;

(iii) if $A_{n} \nearrow A$ and $A_{n}, A \in \mathcal{B}\left(R^{n}\right), n \in N$, then $x\left(A_{n}\right) \nearrow x(A)$.

Similarly as in the classical case the following theorem can be proved ( $[7,13])$.

Theorem 2.7. Let $x: \mathcal{B}(R) \longrightarrow \mathcal{F}$ be an IF-observable, $\mathbf{m}: \mathcal{F} \longrightarrow[0,1]$ be an IF-state. Define the mapping $\mathbf{m}_{x}: \mathcal{B}(R) \longrightarrow[0,1]$ by the formula

$$
\mathbf{m}_{x}(C)=\mathbf{m}(x(C))
$$

Then $\mathbf{m}_{x}: \mathcal{B}(R) \longrightarrow[0,1]$ is a probability measure.

\section{Joint IF-observable, function of several IF-observables and $\mathcal{P}$-almost everywhere convergence}

In [6] we introduced the notion of product operation on the family of IF-events $\mathcal{F}$ and showed an example of this operation.

Definition 3.1. We say that a binary operation $\cdot$ on $\mathcal{F}$ is product if it satisfying the following conditions:

(i) $\left(1_{\Omega}, 0_{\Omega}\right) \cdot\left(a_{1}, a_{2}\right)=\left(a_{1}, a_{2}\right)$ for each $\left(a_{1}, a_{2}\right) \in \mathcal{F}$; 
(ii) The operation - is commutative and associative;

(iii) If $\left(a_{1}, a_{2}\right) \odot\left(b_{1}, b_{2}\right)=\left(0_{\Omega}, 1_{\Omega}\right)$ and $\left(a_{1}, a_{2}\right),\left(b_{1}, b_{2}\right) \in \mathcal{F}$, then

$$
\left(c_{1}, c_{2}\right) \cdot\left(\left(a_{1}, a_{2}\right) \oplus\left(b_{1}, b_{2}\right)\right)=\left(\left(c_{1}, c_{2}\right) \cdot\left(a_{1}, a_{2}\right)\right) \oplus\left(\left(c_{1}, c_{2}\right) \cdot\left(b_{1}, b_{2}\right)\right)
$$

and

$$
\left(\left(c_{1}, c_{2}\right) \cdot\left(a_{1}, a_{2}\right)\right) \odot\left(\left(c_{1}, c_{2}\right) \cdot\left(b_{1}, b_{2}\right)\right)=\left(0_{\Omega}, 1_{\Omega}\right)
$$

for each $\left(c_{1}, c_{2}\right) \in \mathcal{F}$;

(iv) If $\left(a_{1 n}, a_{2 n}\right) \searrow\left(0_{\Omega}, 1_{\Omega}\right),\left(b_{1 n}, b_{2 n}\right) \searrow\left(0_{\Omega}, 1_{\Omega}\right)$ and $\left(a_{1 n}, a_{2 n}\right),\left(b_{1 n}, b_{2 n}\right) \in \mathcal{F}$, then $\left(a_{1 n}, a_{2 n}\right) \cdot\left(b_{1 n}, b_{2 n}\right) \searrow\left(0_{\Omega}, 1_{\Omega}\right)$.

In the following theorem is the example of product operation for IF-events.

Theorem 3.2. The operation · defined by

$$
\left(x_{1}, y_{1}\right) \cdot\left(x_{2}, y_{2}\right)=\left(x_{1} \cdot x_{2}, y_{1}+y_{2}-y_{1} \cdot y_{2}\right)
$$

for each $\left(x_{1}, y_{1}\right),\left(x_{2}, y_{2}\right) \in \mathcal{F}$ is product operation on $\mathcal{F}$.

Proof. In [6, Theorem 1].

In [11] B. Riečan defined the notion of a joint IF-observable and he proved its existence.

Definition 3.3. Let $x, y: \mathcal{B}(R) \rightarrow \mathcal{F}$ be two IF-observables. The joint IF-observable of the IF-observables $x$, y is a mapping $h: \mathcal{B}\left(R^{2}\right) \rightarrow \mathcal{F}$ satisfying the following conditions:

(i) $h\left(R^{2}\right)=\left(1_{\Omega}, 0_{\Omega}\right), h(\emptyset)=\left(0_{\Omega}, 1_{\Omega}\right) ;$

(ii) if $A, B \in \mathcal{B}\left(R^{2}\right)$ and $A \cap B=\emptyset$, then $h(A \cup B)=h(A) \oplus h(B)$ and $h(A) \odot h(B)=\left(0_{\Omega}, 1_{\Omega}\right)$;

(iii) if $A, A_{1}, \ldots \in \mathcal{B}\left(R^{2}\right)$ and $A_{n} \nearrow A$, then $h\left(A_{n}\right) \nearrow h(A)$;

(iv) $h(C \times D)=x(C) \cdot y(D)$ for each $C, D \in \mathcal{B}(R)$.

Theorem 3.4. For each two IF-observables $x, y: \mathcal{B}(R) \rightarrow \mathcal{F}$ there exists their joint IF-observable.

Proof. In [11, Theorem 3.3].

Remark 3.5. The joint IF-observable of IF-observables $x, y$ from Definition 3.3 is two-dimensional IF-observable.

If we have several IF-observables and a Borel measurable function, we can define the IF-observable, which is the function of several IF-observables. About this says the following definition. 
Definition 3.6. Let $x_{1}, \ldots, x_{n}: \mathcal{B}(R) \rightarrow \mathcal{F}$ be IF-observables, $h_{n}$ their joint IF-observable and $g_{n}: R^{n} \rightarrow R$ a Borel measurable function. Then we define the IF-observable $g_{n}\left(x_{1}, \ldots, x_{n}\right): \mathcal{B}(R) \rightarrow \mathcal{F}$ by the formula

$$
g_{n}\left(x_{1}, \ldots, x_{n}\right)(A)=h_{n}\left(g_{n}^{-1}(A)\right) .
$$

for each $A \in \mathcal{B}(R)$.

There exists connection between $\mathcal{P}$-almost everywhere convergence and $\mathbf{m}$-almost everywhere convergence (see [3]).

Theorem 3.7. A sequence $\left(x_{n}\right)_{n}$ of an IF-observables converges $\mathcal{P}$-almost everywhere to 0 if and only if it converges $\mathcal{P}^{b}$-almost everywhere and $\mathcal{P}^{\sharp}$-almost everywhere to 0 .

Proof. In [3, Theorem 5]

\section{Conditional IF-probability and martingale convergence theorem using IF-state}

In [13] B. Riečan defined the conditional probability for IF-case with respect to the IF-state. He was inspired by classical case.

Definition 4.1. Let $y: \mathcal{B}(R) \rightarrow \mathcal{F}$ be an IF-observable, $\mathbf{A} \in \mathcal{F}$. Then the conditional IFprobability $\mathbf{p}(\mathbf{A} \mid y)=f$ is a Borel measurable function (i.e. $B \in \mathcal{B}(R) \Longrightarrow f^{-1}(B) \in \mathcal{B}(R)$ ) such that

$$
\int_{B} \mathbf{p}(\mathbf{A} \mid y) d \mathbf{m}_{y}=\mathbf{m}(\mathbf{A} \cdot y(B))
$$

for each $B \in \mathcal{B}(R)$.

In paper [4] we proved the properties of the conditional IF-probability and the martingale convergence theorem in this case.

Theorem 4.2. Let $\mathcal{F}$ be family of IF-events, $\mathbf{A} \in \mathcal{F}, y: \mathcal{B}(R) \rightarrow \mathcal{F}$ be an IF-observable. Then $\mathbf{p}(\mathbf{A} \mid y)$ has the following properties:

(i) $\mathbf{p}\left(\left(0_{\Omega}, 1_{\Omega}\right) \mid y\right)=0, \mathbf{p}\left(\left(1_{\Omega}, 0_{\Omega}\right) \mid y\right)=1 \mathbf{m}_{y}$-almost everywhere;

(ii) $0 \leq \mathbf{p}(\mathbf{A} \mid y) \leq 1 \mathbf{m}_{y}$-almost everywhere;

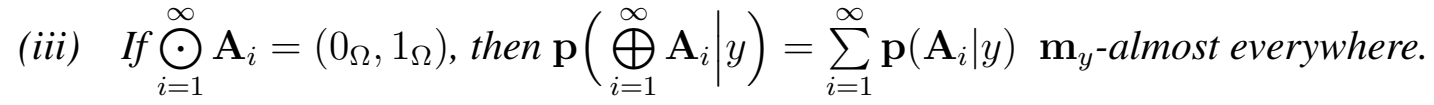

(iv) If $\mathbf{A}_{n} \nearrow \mathbf{A}$, then $\mathbf{p}\left(\mathbf{A}_{n} \mid y\right) \nearrow \mathbf{p}(\mathbf{A} \mid y) \mathbf{m}_{y}$-almost everywhere.

Proof. In [4, Theorem 5]

Theorem 4.3. (Martingale convergence theorem) Let $\mathcal{F}$ be a family of IF-events with product ., $\mathbf{A} \in \mathcal{F}, y: \mathcal{B}(R) \rightarrow \mathcal{F}$ be an IF-observable, $g, g_{n}: R \rightarrow R(n=1,2, \ldots)$ be the Borel measurable functions such that $g_{n}^{-1}(\mathcal{B}(R)) \nearrow g^{-1}(\mathcal{B}(R))$. Then

$$
\mathbf{p}\left(\mathbf{A} \mid y \circ g_{n}^{-1}\right) \rightarrow \mathbf{p}\left(\mathbf{A} \mid y \circ g^{-1}\right) \quad \mathbf{m}_{y \circ g^{-1}}-\text { almost everywhere. }
$$

Proof. In [4, Theorem 6]. 


\section{Conditional IF-probability and martingale convergence theorem using IF-probability}

In this section we formulate modification of the conditional probability and the martingale convergence theorem with respect to the IF-probability in intuitionistic fuzzy case.

Definition 5.1. Let $y: \mathcal{B}(R) \rightarrow \mathcal{F}$ be an IF-observable, $\mathbf{A} \in \mathcal{F}$ and $\mathcal{P}=\left[\mathcal{P}^{b}, \mathcal{P}^{\sharp}\right.$ be an IF-probability. Then a function $\mathbf{p}(\mathbf{A} \mid y): \mathcal{B}(R) \rightarrow \mathcal{J}$ is the conditional IF-probability if there exist the Borel measurable functions $\mathbf{p}^{b}(\mathbf{A} \mid y), \mathbf{p}^{\sharp}(\mathbf{A} \mid y): R \rightarrow R$ such that

$$
\left[\int_{B} \mathbf{p}^{b}(\mathbf{A} \mid y) d \mathcal{P}_{y}^{b}, \int_{B} \mathbf{p}^{\sharp}(\mathbf{A} \mid y) d \mathcal{P}_{y}^{\sharp}\right]=\mathcal{P}(\mathbf{A} \cdot y(B))
$$

for each $B \in \mathcal{B}(R)$. There $\mathbf{p}(\mathbf{A} \mid y)=\left[\mathbf{p}^{b}(\mathbf{A} \mid y), \mathbf{p}^{\sharp}(\mathbf{A} \mid y)\right]$.

Now we show the connection between the conditioning with respect to an IF-state and the conditioning with respect to an IF-probability.

Theorem 5.2. Let $y: \mathcal{B}(R) \rightarrow \mathcal{F}$ be an IF-observable, $\mathbf{A} \in \mathcal{F}$ and $\mathcal{P}=\left[\mathcal{P}^{b}, \mathcal{P}^{\sharp}\right]$ be an IF-probability. The function $\mathbf{p}(\mathbf{A} \mid y): \mathcal{B}(R) \rightarrow \mathcal{J}$ is the conditional IF-probability with respect to the IF-probability $\mathcal{P}$, if and only if the functions $\mathbf{p}^{b}(\mathbf{A} \mid y), \mathbf{p}^{\sharp}(\mathbf{A} \mid y): R \rightarrow R$ are the conditional IF-probabilities with respect to the IF-states $\mathcal{P}^{b}, \mathcal{P}^{\sharp}$ respectively.

Proof. Let $y: \mathcal{B}(R) \rightarrow \mathcal{F}$ be an IF-observable, $\mathbf{A} \in \mathcal{F}$ and $\mathcal{P}=\left[\mathcal{P}^{b}, \mathcal{P}^{\sharp}\right]$ be an IF-probability. " $\Rightarrow$ " Let the function $\mathbf{p}(\mathbf{A} \mid y): \mathcal{B}(R) \rightarrow \mathcal{J}$ is the conditional IF-probability with respect to the IF-probability $\mathcal{P}$. Then by Definition 5.1 there exists the Borel measurable functions $\mathbf{p}^{\mathrm{b}}(\mathbf{A} \mid y), \mathbf{p}^{\sharp}(\mathbf{A} \mid y): R \rightarrow R$ such that

$$
\left[\int_{B} \mathbf{p}^{b}(\mathbf{A} \mid y) d \mathcal{P}_{y}^{b}, \int_{B} \mathbf{p}^{\sharp}(\mathbf{A} \mid y) d \mathcal{P}_{y}^{\sharp}\right]=\mathcal{P}(\mathbf{A} \cdot y(B))
$$

for each $B \in \mathcal{B}(R)$. Using Theorem 2.4 we have

$$
\left[\int_{B} \mathbf{p}^{b}(\mathbf{A} \mid y) d \mathcal{P}_{y}^{b}, \int_{B} \mathbf{p}^{\sharp}(\mathbf{A} \mid y) d \mathcal{P}_{y}^{\sharp}\right]=\mathcal{P}(\mathbf{A} \cdot y(B))=\left[\mathcal{P}^{b}(\mathbf{A} \cdot y(B)), \mathcal{P}^{\sharp}(\mathbf{A} \cdot y(B))\right] .
$$

Hence

$$
\begin{aligned}
& \int_{B} \mathbf{p}^{b}(\mathbf{A} \mid y) d \mathcal{P}_{y}^{b}=\mathcal{P}^{b}(\mathbf{A} \cdot y(B)), \\
& \int_{B} \mathbf{p}^{\sharp}(\mathbf{A} \mid y) d \mathcal{P}_{y}^{\sharp}=\mathcal{P}^{\sharp}(\mathbf{A} \cdot y(B))
\end{aligned}
$$

for each $B \in \mathcal{B}(R)$. Therefore by Definition $4.1 \mathbf{p}^{\mathrm{b}}(\mathbf{A} \mid y), \mathbf{p}^{\sharp}(\mathbf{A} \mid y): R \rightarrow R$ are the conditional IF-probabilities with respect to the IF-states $\mathcal{P}^{b}, \mathcal{P}^{\sharp}$ respectively.

“ $\Leftarrow$ " It can be proved in the opposite direction as in " $\Rightarrow$ ".

Theorem 5.3. Let $\mathcal{F}$ be family of IF-events, $\mathbf{A} \in \mathcal{F}, y: \mathcal{B}(R) \rightarrow \mathcal{F}$ be an IF-observable. Then $\mathbf{p}(\mathbf{A} \mid y): \mathcal{B}(R) \rightarrow \mathcal{J}$ has the following properties: 
(i) $\mathbf{p}\left(\left(0_{\Omega}, 1_{\Omega}\right) \mid y\right)=[0,0]=0, \mathbf{p}\left(\left(1_{\Omega}, 0_{\Omega}\right) \mid y\right)=[1,1]=1 \mathcal{P}_{y}$-almost everywhere;

(ii) $0 \leq \mathbf{p}(\mathbf{A} \mid y) \leq 1 \mathcal{P}_{y}$-almost everywhere;

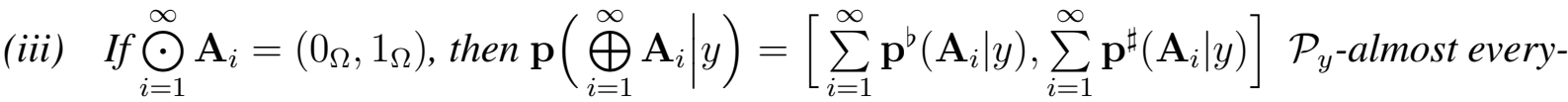
where.

(iv) If $\mathbf{A}_{n} \nearrow \mathbf{A}$, then $\mathbf{p}\left(\mathbf{A}_{n} \mid y\right) \nearrow \mathbf{p}(\mathbf{A} \mid y) \mathcal{P}_{y}$-almost everywhere.

Proof. The proof is straightforward using Theorem 5.2, Theorem 4.2 and Theorem 3.7.

Theorem 5.4 (Martingale convergence theorem). Let $\mathcal{F}$ be a family of IF-events with product -, $\mathbf{A} \in \mathcal{F}, y: \mathcal{B}(R) \rightarrow \mathcal{F}$ be an IF-observable, $\mathcal{P}$ be an IF-probability, $g, g_{n}: R \rightarrow R$ $(n=1,2, \ldots)$ be the Borel measurable functions such that $g_{n}^{-1}(\mathcal{B}(R)) \nearrow g^{-1}(\mathcal{B}(R))$. Then

$$
\mathbf{p}\left(\mathbf{A} \mid y \circ g_{n}^{-1}\right) \rightarrow \mathbf{p}\left(\mathbf{A} \mid y \circ g^{-1}\right) \quad \mathcal{P}_{y \circ g^{-1}} \text { - almost everywhere. }
$$

Proof. By Definition 5.1 and Theorem 5.2 we have

$$
\begin{aligned}
& \mathbf{p}\left(\mathbf{A} \mid y \circ g_{n}^{-1}\right)=\left[\mathbf{p}^{\mathrm{b}}\left(\mathbf{A} \mid y \circ g_{n}^{-1}\right), \mathbf{p}^{\sharp}\left(\mathbf{A} \mid y \circ g_{n}^{-1}\right)\right], \\
& \mathbf{p}\left(\mathbf{A} \mid y \circ g^{-1}\right)=\left[\mathbf{p}^{\mathrm{b}}\left(\mathbf{A} \mid y \circ g^{-1}\right), \mathbf{p}^{\sharp}\left(\mathbf{A} \mid y \circ g^{-1}\right)\right],
\end{aligned}
$$

where $\mathbf{p}^{b}\left(\mathbf{A} \mid y \circ g_{n}^{-1}\right), \mathbf{p}^{\sharp}\left(\mathbf{A} \mid y \circ g_{n}^{-1}\right): R \rightarrow R$ and $\mathbf{p}^{b}\left(\mathbf{A} \mid y \circ g^{-1}\right), \mathbf{p}^{\sharp}\left(\mathbf{A} \mid y \circ g^{-1}\right): R \rightarrow R$ are the conditional IF-probabilities with respect to the IF-states $\mathcal{P}^{b}, \mathcal{P}^{\sharp}$ respectively.

Then using Theorem 4.3 we obtain that

$$
\begin{array}{ll}
\mathbf{p}^{b}\left(\mathbf{A} \mid y \circ g_{n}^{-1}\right) \rightarrow \mathbf{p}^{b}\left(\mathbf{A} \mid y \circ g^{-1}\right) & \mathcal{P}_{y \circ g^{-1}}^{b}-\text { almost everywhere, } \\
\mathbf{p}^{\sharp}\left(\mathbf{A} \mid y \circ g_{n}^{-1}\right) \rightarrow \mathbf{p}^{\sharp}\left(\mathbf{A} \mid y \circ g^{-1}\right) & \mathcal{P}_{y \circ g^{-1}}^{\sharp}-\text { almost everywhere. }
\end{array}
$$

Therefore by Theorem 3.7 we have

$$
\begin{aligned}
\mathbf{p}\left(\mathbf{A} \mid y \circ g_{n}^{-1}\right) & =\left[\mathbf{p}^{\mathrm{b}}\left(\mathbf{A} \mid y \circ g_{n}^{-1}\right), \mathbf{p}^{\sharp}\left(\mathbf{A} \mid y \circ g_{n}^{-1}\right)\right] \rightarrow\left[\mathbf{p}^{\mathrm{b}}\left(\mathbf{A} \mid y \circ g^{-1}\right), \mathbf{p}^{\sharp}\left(\mathbf{A} \mid y \circ g^{-1}\right)\right] \\
& =\mathbf{p}\left(\mathbf{A} \mid y \circ g^{-1}\right) \quad \mathcal{P}_{y \circ g^{-1} \text {-almost everywhere. }}
\end{aligned}
$$

\section{Conclusion}

The paper is concerned in the probability theory on intuitionistic fuzzy sets. We defined the conditional intuitionistic fuzzy probability induced by an intuitionistic fuzzy probability and we proved its properties. We showed the connection between conditioning with respect to the intuitionistic fuzzy probability and conditioning with respect to the intuitionistic fuzzy state. We formulated and proved the martingale convergence theorem for the conditional intuitionistic fuzzy probability with respect to the intuitionistic fuzzy probability, too. 


\section{References}

[1] Atannasov, K. (1999). Intuitionistic Fuzzy Sets: Theory and Applications, Springer Physica Verlag, Heindelberg.

[2] Atanassov, K. T. (2012). On Intuitionistic Fuzzy Sets, Springer, Berlin.

[3] Čunderlíková, K. (2019). Intuitionistic fuzzy probability and almost everywhere convergence, Proceedings of IWIFSGN 2019, 24-25 October 2019, Warsaw, Poland (accepted).

[4] Čunderlíková, K. (2020). Martingale convergence theorem for the conditional intuitionistic fuzzy probability, Mathematics, 8 (10), 1707, 1-10.

[5] Grzegorzewski, P., \& Mrówka, E. (2002). Probability of intuistionistic fuzzy events, Soft Metods in Probability, Statistics and Data Analysis, P. Grzegorzewski et al. eds., Physica Verlag, New York, 105-115.

[6] Lendelová, K. (2006). Conditional IF-probability, Advances in Soft Computing: Soft Methods for Integrated Uncertainty Modelling, 275-283.

[7] Lendelová, K., \& Riečan, B. (2004). Weak law of large numbers for IF-events, Current Issues in Data and Knowledge Engineering, Bernard De Baets et al. eds., EXIT, Warszawa, 309-314.

[8] Riečan, B. (2003). A descriptive definition of the probability on intuitionistic fuzzy sets, EUSFLAT '2003, M. Wagenecht, R. Hampet eds., Zittau-Goerlitz Univ. Appl. Sci., $263-$ 266.

[9] Riečan, B. (2005). On the probability on IF-sets and MV-algebras, Notes on Intuitionistic Fuzzy Sets, 11 (6), 21-25.

[10] Riečan, B. (2006). On a problem of Radko Mesiar: general form of IF-probabilities, Fuzzy Sets and Systems, 152, 1485-1490.

[11] Riečan, B. (2006) On the probability and random variables on IF events, Applied Artifical Intelligence, Proc. 7th FLINS Conf. Genova, D. Ruan et al. eds., 138-145.

[12] Riečan, B. (2007). Probability theory on intuitionistic fuzzy events, Algebraic and Prooftheoretic aspects of Non-classical Logics, Papers in honour of Daniele Mundici's 60th birthday, Lecture Notes in Computer Science, Vol. 4460.

[13] Riečan, B. (2012). Analysis of fuzzy logic models, Intelligent Systems (V. Koleshko ed.), INTECH, 219-244.

[14] Riečan, B., \& Neubrunn, T. (1997). Integral, Measure, and Ordering, Kluwer Academic Publishers, Dordrecht and Ister Science, Bratislava. 